\title{
Nonlinear self-trapping of broad beams in defocusing lithium niobate waveguide arrays
}

\author{
Francis H. Bennet, ${ }^{1}$ Tristram J. Alexander, ${ }^{1}$ Franz Haslinger, ${ }^{1}$ \\ Arnan Mitchell, ${ }^{2}$ Dragomir N. Neshev, ${ }^{1}$ and Yuri S. Kivshar ${ }^{1}$ \\ ${ }^{1}$ Nonlinear Physics Centre, Research School of Physics and Engineering, \\ Australian National University, Canberra, ACT 0200 Australia \\ ${ }^{2}$ School of Electrical and Computer Engineering, RMIT, Melbourne Vic 3001, Australia
}

\begin{abstract}
We demonstrate experimentally the localization of broad beams in defocusing waveguide arrays. Unlike gap solitons, these novel localized states have an arbitrary width defined by the size of the input beam while independent on nonlinearity.
\end{abstract}

(C) 2010 Optical Society of America

OCIS codes: (190.4420) Nonlinear optics, transverse effects in; (190.5940) Self-action effects

The coherent transport of nonlinear waves is essential in many phenomena in nature. In contrast to linear waves, the physics becomes complex when wave interactions start to play a role, making laboratory studies difficult. As such, many of the fundamental physical effects of nonlinear waves have been first studied in optics, where the advent of the laser as an intense coherent light source gave rise to the field of nonlinear optics. The recent experimental developments in Bose-Einstein condensates (BEC) opened the way for many analogous experiments with intense sources of coherent matter waves.

A few years ago Anker et al. [1] reported the experimental observation of nonlinear self-trapping of Bose-condensed atoms in stationary periodic potentials. This trapping effect due to interaction between condensed atoms is manifested as a change from the diffusive regime, characterized by an expansion of the condensate, to the nonlinearity dominated self-trapping regime, where the initial expansion stops and the width of a matter-wave packet remains finite. This observation abrogates a seemingly obvious analogy between nonlinear optics and matter wave physics, because this type of nonlinearity-induced self-trapping in the presence of repulsive interaction has never been observed in optics. In this paper we report on the first experimental observation of nonlinear self-trapping of broad optical beams with zero transverse momentum in an array of defocusing optical waveguides. Our results not only provide an optical analogue of the self-trapping effect of Bose-condensed atoms [1-3], but also reveal the important features of such localized states not observed earlier. In particular, we demonstrate that unlike conventional gap solitons observed in defocusing photonic lattices [4], the spatial extent of such self-trapped states is controlled by the width of the input excitation remaining independent on power.

We examine the excitation of such localized Bloch waves from a Gaussian input. Figure 1 summarizes the numerical results on the Bloch wave excitation for different values of the optical nonlinearity. As shown in Fig. 1(a), for weak defocusing nonlinearity the beam undergoes rapid spreading (faster than linear diffraction). However as the nonlinearity is increased this spreading is suddenly halted and the beam localizes with a width of the order of the width of the input beam. As is evident in the comparison of Figs. 1(b,c) the signature of the truncated nonlinear Bloch wave is the sharp intensity dropoff in the wings of the beam. Additional features visible in Figs. 1(a,b) are the strong intensity modulations occurring within the localized state. The large diffraction coefficient leads to long-lived nonlinear excitations of the truncated nonlinear Bloch wave, which despite the strong modifications of the intensity profile do not lead to decay of the localized state. Most
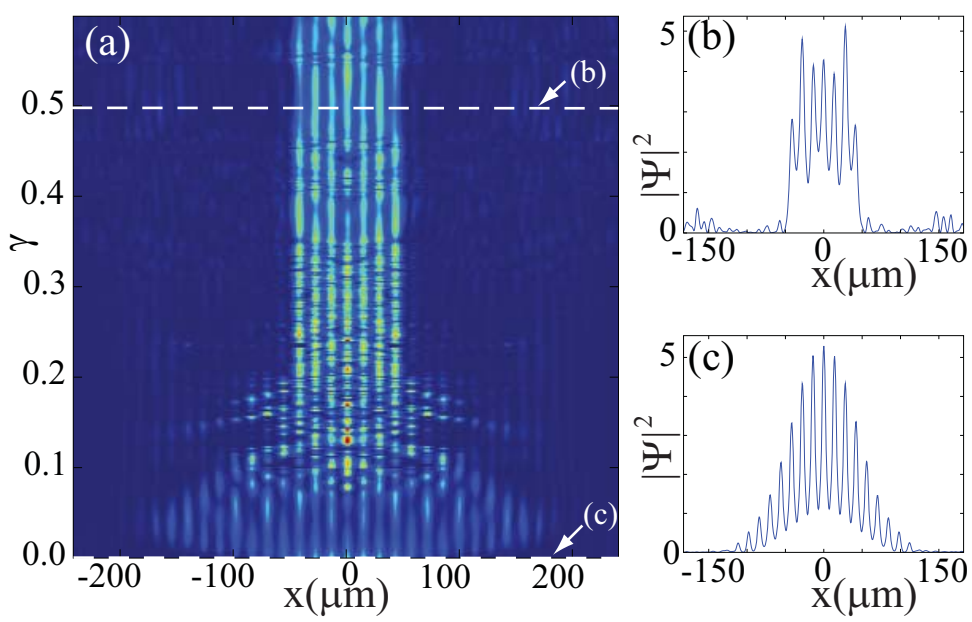

Fig. 1. (a) Dependence of output intensity on nonlinearity $\gamma$. Initial beam spreading is arrested at a critical value of the nonlinearity. (b,c) Example output beam profiles (as marked in (a)). importantly, Fig. 1(a) shows that above a certain threshold for the optical nonlinearity $(\gamma \approx 0.15)$, the width of the 
localized state remains practically independent on the input beam power.

Experimentally, we test the excitation of truncated Bloch waves by employing the defocusing nonlinearity of a $\mathrm{LiNbO}_{3}$ waveguide array $(6 \mathrm{~cm}$ long, fabricated by Titanium indiffusion). Due to the slow nature of the photovoltaic nonlinear response in $\mathrm{LiNbO}_{3}$, the nonlinear index change increases slowly with time under a constant input laser power. Since the time-scale of this index change is of the order of several minutes, the time dependence of the beam output intensity profile can be effectively mapped to the dependence on the nonlinear coefficient $\gamma$.

We excite the array with a broad Gaussian laser beam at $532 \mathrm{~nm}$. The input beam [Fig. 2(a)] is elliptically shaped by a cylindrical lens $(f=50 \mathrm{~mm})$ before a (20x) focusing objective. We monitor the beam output intensity profile with time for a typical input power of $1 \mathrm{~mW}$ and measure the variation of the beam width. The width of the output beam (Fig. 2, main panel) is determined as the size of the area which contains $50 \%$ of the output light power. This allows us to filter out any noise in the diffraction pattern while maintaining a high degree of accuracy. Error bars are calculated as the asymmetry of the output profile with respect to the center of the input beam.

The linear diffraction in the array causes the beam to spread out and occupy nearly twice the number of waveguides in comparison to the input beam [Fig. 2(b)]. Upon increase of the nonlinearity with time, we observe an initial defocusing of the
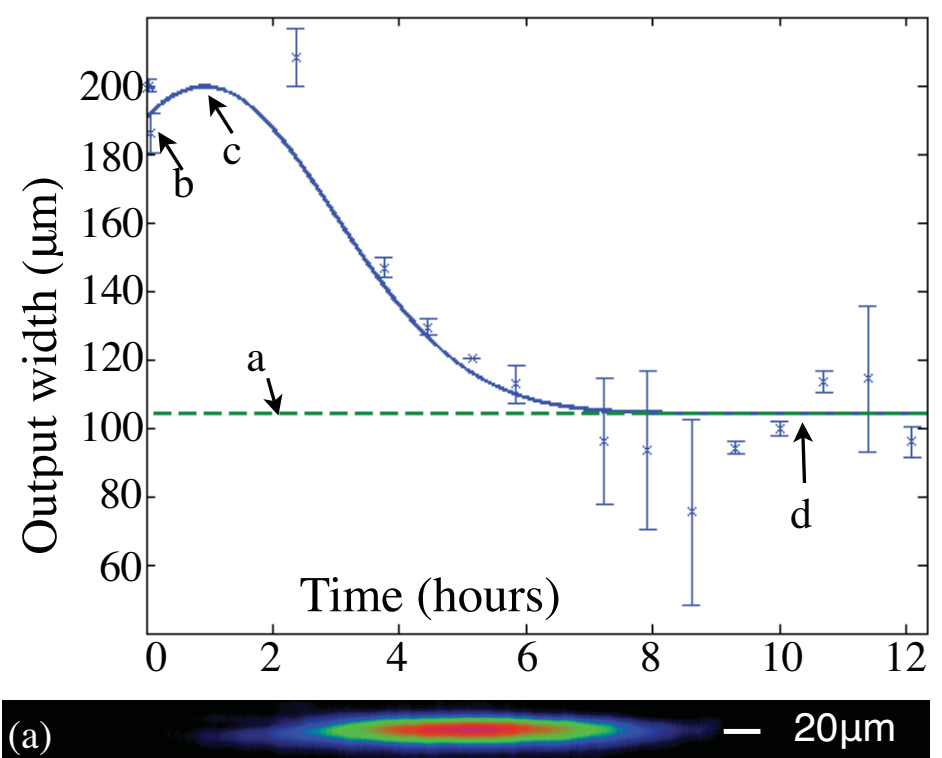

(b) (c) (d)

Fig. 2. Output beam width vs. time (nonlinear index change). Dashed line $-75 \%$ of the input beam width. (a) Input beam profile; (b-d) Output beam profiles at different time (as marked in the graph). Beam power, $1 \mathrm{~mW}$. beam $[(\mathrm{c})]$ resulting from the weak negative nonlinearity. As the exposure time increases and the nonlinearity grows, the beam experiences gradual confinement and reduces its width [(d)]. The dependence of the beam width with time shows that after the initial expansion, the beam localizes and the width remains essentially constant with nonlinearity. This localized state has a width equal to the width of the input beam containing $75 \%$ of the power, where the remaining $25 \%$ have been lost in radiation. Small oscillations around this value are observed at longer times, thus matching the numerical predictions in Fig. 1. Additional proof that the nonlinear localization is inside the Bragg reflection gap is seen in the appearance of spatial frequencies at the edges of the Brillouin zone.

A unique property of the optical system is the ability to control independently the width and the power of the input beam. This control allows us to test the new feature of nonlinear Bloch wave localization - that it is parameterized by the input beam width rather than by the input power. For this purpose the input beam width in our experiment is reduced by half. Using such narrow beams, we can again identify similar behavior of the output beam evolution. First we observe the initial beam defocusing with increase of the nonlinearity, while at longer times the beam confines to a narrower localized state equal to the width of the input beam containing about $65 \%$ of the total input power.

In conclusion, we have observed nonlinear self-trapping of broad optical beams in defocusing waveguide arrays. We have revealed that these novel types of spatially localized modes can have an arbitrary width defined by the input beam, while the width is practically independent of nonlinearity. We believe that the unique properties of the truncated nonlinear Bloch states will encourage their observation in other physical systems.

\section{References}

1. Th. Anker, M. Albiez, R. Gati, S. Hunsmann, B. Eiermann, A. Trombettoni, and M. K. Oberthaler, Phys. Rev. Lett. 94, 020403 (2005).

2. T. J. Alexander, E. A. Ostrovskaya, and Yu. S. Kivshar, Phys. Rev. Lett. 96, 040401 (2006).

3. J. Wang, J. Yang, T. J. Alexander, and Yu. S. Kivshar, Phys. Rev. A 79, 043610 (2009).

4. M. Matuszewski, C. R. Rosberg, D. N. Neshev, A. A. Sukhorukov, A. Mitchell, M. Trippenbach, M. A. Austin, W. Krolikowski, and Yu. S. Kivshar, Opt. Express 14, 254 (2006). 